\title{
The IFAE/UAB Raman LIDAR for the CTA-North
}

\author{
Markus Gaug ${ }^{1, *}$, Oscar Blanch ${ }^{2}$, Merve Sıdıka Çolak ${ }^{2}$, Michele Doro ${ }^{3}$, Lluis Font $^{1}$, Scott Griffiths ${ }^{1, * *}$, Camilla Maggio ${ }^{1}$, \\ Manel Martinez ${ }^{2}$, Òscar Martinez ${ }^{2}$, Pere Munar-Adrover ${ }^{1}$, and Vania Da Deppo ${ }^{5}$ for the CTA Consortium \\ ${ }^{1}$ Unitat de Física de les Radiacions, Departament de Física, and CERES-IEEC, \\ Universitat Autònoma de Barcelona, E-08193 Bellaterra, Spain \\ ${ }^{2}$ Institut de Física d'Altes Energies (IFAE), The Barcelona Institute of Science and Technology (BIST), \\ E-08193 Bellaterra (Barcelona), Spain \\ ${ }^{3}$ Università di Padova and INFN, I-35131 Padova, Italy \\ ${ }^{4}$ CNR-Institute for Photonics and Nanotechnologies UOS Padova LUXOR, Via Trasea 7, 35131 Padova, Italy
}

\begin{abstract}
The IFAE/UAB Raman LIDAR project aims to develop a Raman LIDAR suitable for the online atmospheric calibration of the CTA. Requirements for such a solution include the ability to characterize aerosol extinction to distances of more than $20 \mathrm{~km}$ with an accuracy better than $5 \%$, within time scales of less than one minute. The Raman LIDAR consists therefore of a large $1.8 \mathrm{~m}$ mirror and a powerful pulsed Nd-YAG laser. A liquid light-guide collects the light at the focal plane and transports it to the readout system. An in-house built polychromator has been characterized thoroughly with respect to its capability to separate efficiently the different wavelengths $(355 \mathrm{~nm}, 387 \mathrm{~nm}, 532 \mathrm{~nm}$ and $607 \mathrm{~nm})$. It was found to operate according to specifications, particularly that light leakage from the elastic channels $(532 \mathrm{~nm}$ and $355 \mathrm{~nm}$ ) into the much dimmer Raman channels ( $387 \mathrm{~nm}$ and $607 \mathrm{~nm}$ ) could be excluded to less than $2 \times 10^{-7}$. We present here the status of the integration and commissioning of this solution and plans for the near future. After a one-year test period at the Observatorio del Roque de los Muchachos, an in-depth evaluation of this and the solutions adopted by a similar project developed by the LUPM, Montpellier, will lead to a final Raman LIDAR proposed to be built for both CTA sites.
\end{abstract}

\section{Introduction}

The Cherenkov Telescope Array (CTA) [1] will observe cosmic $\gamma$-rays from few tens of $\mathrm{GeV}$ to several hundreds of $\mathrm{TeV}$ with greatly improved sensitivity, angular and energy resolution over current experiments [2]. Such improvements must come along with a significant reduction of systematic uncertainties in energy and flux reconstruction [3], dominated by atmospheric effects [4]. The local troposphere and lower stratosphere must hence be monitored, such that telescope data can be corrected offline before dissemination. The CTA has opted for a combination of two complementary instruments to achieve this goal: A wide-field telescope optimized for stellar photometry $[5,6]$ across a field-of-view as large as that of CTA and a powerful Raman LIDAR [7] capable of characterizing aerosol extinction from ground to about $20 \mathrm{~km}$ a.s.l. during the short time scales on which the CTA telescopes either change observation target or Wobble position [8].

The Institut de Física d'Altes Energies (IFAE) and the Universitat Autònoma de Barcelona (UAB) joined in the IFAE/UAB Raman LIDAR project and designed such LIDARs [9], as well as another group at LUPM, France, tailored to the requirements of CTA: precise characterization of the aerosol extinction over a large dynamic range

\footnotetext{
*e-mail: markus.gaug@uab.cat

** now at Rockwell Automation, Inc.
}

on short time scales using two wavelengths characteristic for the measured Cherenkov light, and their corresponding Stokes lines due to Raman scattering on atmospheric nitrogen. Detailed link-budget simulations [10] have predicted the basic functioning of the chosen solution, but construction of a working prototype has met several difficulties so far, related to the large optics required and the corresponding dimensions of the polychromator [11]. We present here the first commissioning results of the working prototype, demonstrating its basic performance.

\section{The IFAE/UAB Raman LIDAR}

The LIDAR consists of a Q-switched Nd:YAG laser, frequency doubled and tripled to $532 \mathrm{~nm}$ and $355 \mathrm{~nm}$, respectively, and a $1.8 \mathrm{~m}$ diameter parabolic receiving mirror, adopted from the discountinued CLUE experiment [12]. At the telescope focus, an $8 \mathrm{~mm}$ wide liquid light guide (LLG) of type Lumatec Series $300^{1}$ transports the light to a polychromator unit, mounted on the back part of the LIDAR telescope structure. The polychromator separates the four wavelengths and detects the light with four Hamamatsu R11920 photomultiplier tube (PMT) units, identical to the ones used in the CTA Large Size Telescopes (LSTs) cameras [13]. The PMT signals are registered by a com-

\footnotetext{
${ }^{1}$ www.lumatec.de/en/products/liquid-light-guide-series-300/
} 
mercial data acquisition system from LICEL $^{2}$. The laser of type Brilliant from Quantel ${ }^{3}$ is mounted on an $X Y$-table with stepping motors at the side of the telescope structure (see Fig. 1). The beam exits along the optical axis of the main mirror in a coaxial configuration thanks to two $5^{\prime \prime}$ dichroic guiding mirrors (tailored to our needs and produced by Optoprim Italia), which act also as absorbers of the spurious $1064 \mathrm{~nm}$ wavelength, which itself could not be blocked at the laser. Table 1 lists further specifications of the system.

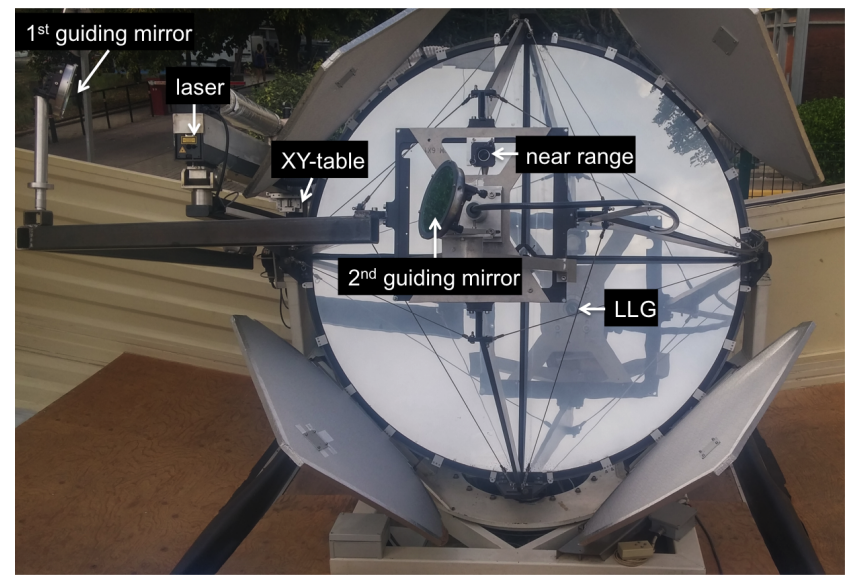

Figure 1. Photo of the IFAE/UAB Raman LIDAR with the laser, the XY-table and the two guiding mirrors. In the reflection of the primary mirror, the focal plane is visible with the liquid light guide (LLG) in its center. Slightly above the second guiding mirror, the near-range receiver is already installed.

\begin{tabular}{ll}
\hline Laser wavelengths & $355 \mathrm{~nm}, 532 \mathrm{~nm}$ \\
Raman wavelengths & $387 \mathrm{~nm}, 607 \mathrm{~nm}$ \\
Energy per pulse & $100 \mathrm{~mJ}, 200 \mathrm{~mJ}$ \\
Pulse duration & $4 \mathrm{~ns}$ \\
Beam divergence & $\approx 1 \mathrm{mrad}$ \\
Mirror area & $2.5 \mathrm{~m}^{2}$ \\
Focal length & $1.8 \mathrm{~m}$ \\
Point spread diameter & $3.3 \mathrm{mrad}(6 \mathrm{~mm})$ \\
Receiver Field of view & $4.4 \mathrm{mrad}(8 \mathrm{~mm})$ \\
Full overlap at & $<150 \mathrm{~m}^{*}$ \\
Number channels & $4^{*}$ \\
Filters width FWHM & $10 \mathrm{~nm}$ \\
Sampling speed & $20 \mathrm{MS} / \mathrm{s}$ \\
Spatial resolution & $7.5 \mathrm{~m}$ \\
\hline
\end{tabular}

Table 1. IFAE/UAB Raman LIDAR specifications. $\dagger 80 \%$ encircled energy

* does not yet include a dedicated near-range optics and readout.

\section{The Polychromator}

The polychromator consists of a series of lenses, dichroic mirrors and interference filters, used to separate efficiently

\footnotetext{
${ }^{2}$ www.licel.com

${ }^{3}$ https://www.quantel-laser.com
}
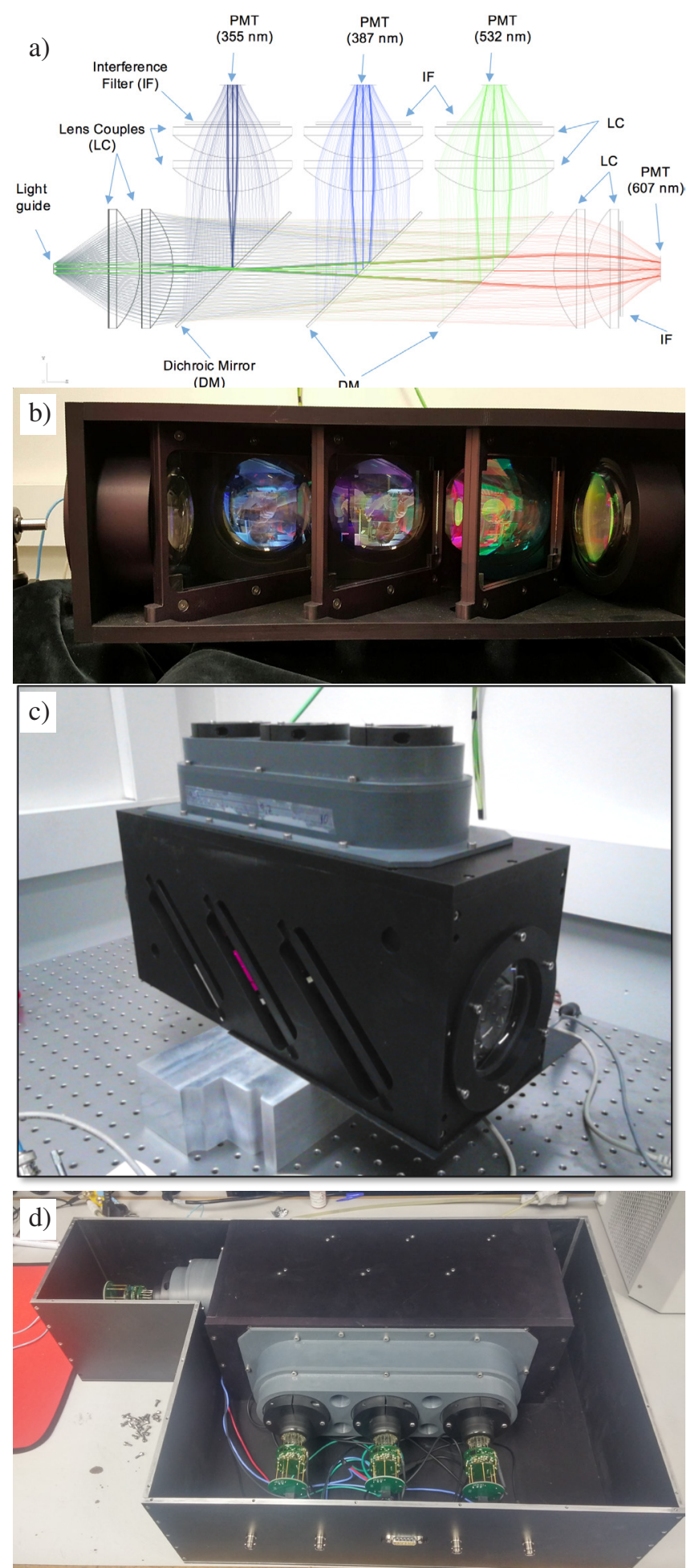

Figure 2. a) Optical design (Zemax $®)$ of the polychromator, b) view from below into the polychromator with the dichroic mirrors visible, c) image of the closed polychromator without housing and PMTs, d) image of the polychromator inside its housing and PMTs mounted.

the four wavelengths of interest and guide the light of each wavelength to its corresponding 1.5" PMT. The polychromator optics were designed in collaboration with the CNR Institute for Photonics and Nanotechnologies in Padova, 
Italy [11] and its mechanics was designed and built at IFAE.

The receiver telescope has a low $f$-number, i.e. 1.0, and a large point spread function, about $6 \mathrm{~mm}$ in diameter. Hence the dimension and the high divergence of the beam exiting the light guide $\left(8 \mathrm{~mm}\right.$ in size and $70^{\circ}$ fieldof-view) have been the main challenges for the polychromator optical design. The chosen optical solution adopts lenses having $100 \mathrm{~mm}$ of diameter hosted inside a box of $760 \times 550 \times 170 \mathrm{~mm}$ (see Fig. 2). The polychromator itself is housed in an anodized aluminum box. Its total mass is about $30 \mathrm{~kg}$. At the beginning of the optical path, the light exiting the liquid light guide is collimated by a Lens Couple (LC), then it is separated in its different components by Dichroic Mirrors (DM), specifically designed for this purpose by Optics Balzers Jena GmbH. After this, in each channel, a second LC focalizes the beam onto the PMTs' photo-cathode. Before reaching the PMTs, the light passes through an interference filter with transmission centered at the desired wavelength and a FWHM of about $10 \mathrm{~nm}$.

The Polychromator has been extensively tested (Figure 3) for correct functioning in the optics lab of IFAE [14]. Among others, possible light leakage has been measured and found to be less than $2 \times 10^{-7}$ for all possible combinations of elastically back-scattered wavelengths leaking into a Raman channel readout of the polychromator.

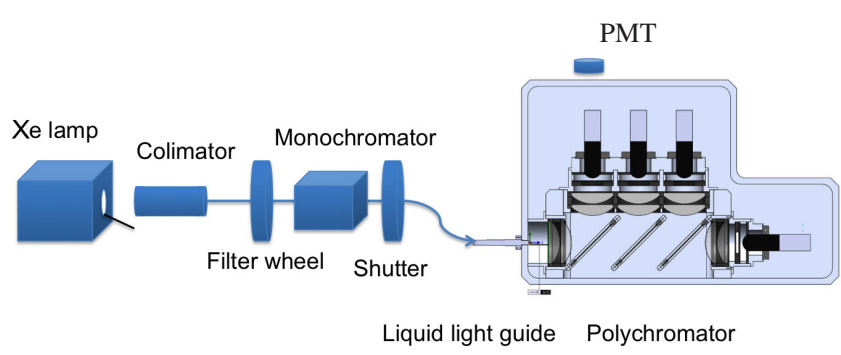

Figure 3. Sketch of the setup for polychromator tests: the collimated light of a stabilized Xe lamp passes first through a broadband filter to discriminate unwanted harmonics of the grating monochromator. A shutter allows to remotely switch on and off the monochromatic light. Up to this stage, the setup is covered by a box (not shown here) to avoid light leakaging out of the system and entering the polychromator from outside. Finally, the light is fed into the very same liquid guide used for the LIDAR and coupled to the polychromator unit. Finally, the PMT current is analyzed by a picoampere-meter. Fast series of measurements with open shutter have been taken, immediately followed by background estimates using the closed shutter, until sufficient statistics have been accumulated.

\section{First commissioning data}

Commissioning data of the integrated system was obtained over several nights, first with greatly reduced laser power for the alignment of the laser beam with the optical axis of the primary mirror. In a later step, data were taken with full laser power after 2 a.m., when the close-by airports of Barcelona and Sabadell close. The LIDAR was aligned first by eye, using the possibility to move the two dichroic guiding mirrors by hand. In a second step, the stepping motors of the laser mount are used, together with one of the two elastic lines connected to an oscilloscope, in order to fine-tune the alignment. Normally, both steps need to be iterated at least once, because the beam may easily move out of the second guiding mirror during fine-tuning. We needed to learn how to align efficiently the LIDAR during several nights, but are now able to align the system in less than one hour, even after starting from a completely misaligned state.

During one night with a sky uniformly covered by layer of high clouds, we tested that the system could correctly image the cloud after moving to different positions, reaching almost horizontal pointing. The alignment remains hence stable under movements of the bulky mirror.
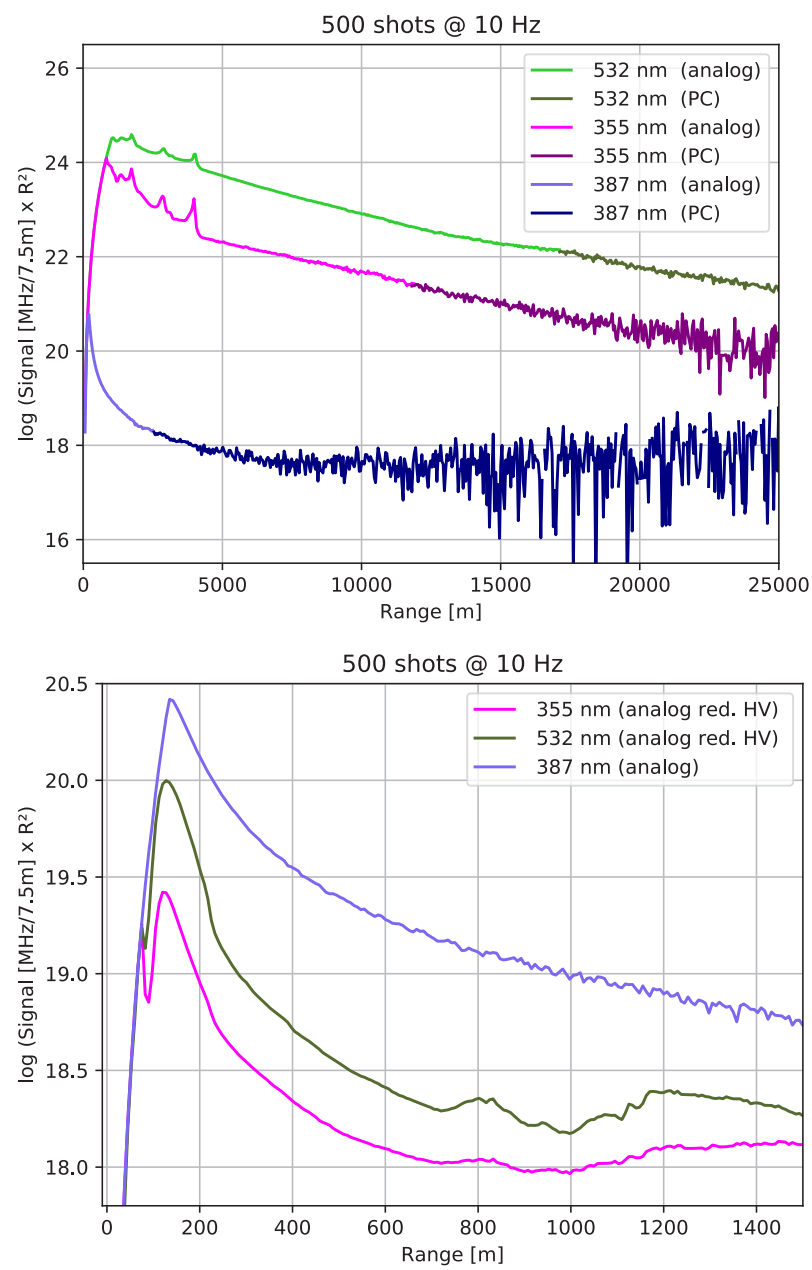

Figure 4. First commissioning signals of the Raman LIDAR at the UAB university campus. Top: The LIDAR has been operated with maximum PMT gain and laser power to demonstrate its long-range behaviour. A $45 \mathrm{~m}$ bin width is used here. Bottom: The LIDAR has been operated with reduced PMT gain for the two elastic lines to demonstrate its short-range behaviour. A $7.5 \mathrm{~m}$ bin width is shown here. The analog part of the signal was "glued" to the photon-counting (PC) part following the prescriptions of $[15,16]$. 
Finally, Figure 4 shows the logarithm of the rangecorrected signals for two configurations of the Raman LIDAR: in the upper part, the full power of the system was tested and the signals are shown up to $25 \mathrm{~km}$ distance, whereas the lower part shows a near-range configuration with the PMT gains of the two elastic lines reduced by a factor of several hundreds to avoid saturation. One can see that the system is easily capable of sensing the atmosphere to further than $25 \mathrm{~km}$ distance within less than a minute. On the near-range side, full overlap is already reached at $125 \mathrm{~m}$, consistent with the theoretical expectation $[17,18]$. Note also the Raman line of $387 \mathrm{~nm}$ saturates the readout below about $150 \mathrm{~m}$ distance.

Tests are still ongoing to understand the currently tooweak Raman line at $607 \mathrm{~nm}$ and to commission a dedicated near-range optics to further increase the dynamic range of the system and to access the altitude range from ground to about $200 \mathrm{~m}$ where most of the aerosols are normally expected [19]. That solution [14] combines a collimator of type RC12SMA-F01 from Thorlabs ${ }^{4}$ with a standard $1.5 \mathrm{~mm}$ fibre $^{5}$. For the moment, the near-range light will be registered by only one PMT, of the same type as those used for the polychromator, in a small "monochromator" box, with just one interference filter for the elastic $532 \mathrm{~nm}$ line.

\section{Conclusions}

The IFAE/UAB Raman LIDAR prototype is now almost complete and has shown to perform as expected for two elastic and one Raman line. Another Raman line at $607 \mathrm{~nm}$ is currently being commissioned as well as a small nearrange solution. Further, easy-to-achieve improvements concern a general cleaning of the mirror, expected to significantly increase its reflectivity, and to increase the laser rate from currently $10 \mathrm{~Hz}$ to $20 \mathrm{~Hz}$. Moreover, we concentrate on further robotization of the system and to achieve compliance with all safety and engineering requirements as well as the communication protocols used by the CTA. Once these steps are completed, the LIDAR will undergo a one-year test at the Observatorio del Roque de los Muchachos, participating in a cross-calibration campaign with an EARLINET-calibrated LIDAR for site characterization [20]. An in-depth evaluation of this and the solutions adopted by a similar project developed by the LUPM, Montpellier, will lead to a final Raman LIDAR proposed as in-kind contribution for the CTA at both sites.

\footnotetext{
${ }^{4}$ www.thorlabs.com/thorproduct.cfm?partnumber=RC12SMA-F01

${ }^{5}$ www.thorlabs.com/thorproduct.cfm? partnumber $=$ FT1500UMT
}

\section{References}

[1] B.S. Acharya et al., Astroparticle Physics 43, 3 (2013)

[2] T. Hassan et al., Astroparticle Physics 93, 76 (2017), 1705.01790

[3] M. Gaug et al., in Proc. SPIE Observatory Operations: Strategies, Processes, and Systems V (2014), Vol. 9149, p. 914945

[4] M. Gaug, in EPJ Web of Conferences (2017), Vol. 144, p. 01003

[5] J. Ebr et al., New developments in aerosol measurements using stellar photometry, in these proceedings (2018)

[6] P. Janeček et al., FRAM telescopes and their measurements of aerosol content at the Pierre Auger $\mathrm{Ob}$ servatory and at future sites of the Cherenkov Telescope Array, in these proceedings (2018)

[7] U. Wandinger, in Lidar Range-Resolved Optical Remote Sensing of the Atmosphere (Springer, 2005), chap. 9, pp. 241-271

[8] M. Doro et al., in Proc. First AtmoHEAD Conference, Saclay (2014), arXiv: 1402.0638

[9] A. López-Oramas et al., in Proc. $33^{\text {rd }}$ ICRC (2013), p. 0210, arXiv:1307.5092

[10] M. Eizmendi, Master's thesis, Universitat Politécnica de Catalunya (UPC) \& Universitat Autònoma de Barcelona (UAB) (2011)

[11] V. Da Deppo et al., in Proc. SPIE Optical Systems Design (2012), Vol. 8550, p. $85501 \mathrm{~V}$

[12] D. Alexandreas et al., NIM A 360, 385 (1995)

[13] T. Toyama et al., NIM A 787, 280 (2015)

[14] O. Calpe Blanch (2017), bachelor thesis, Universitat Autònoma de Barcelona

[15] R.K. Newsom et al., Appl. Opt. 48, 3903 (2009)

[16] D. Lange et al., Revista Boliviana de Física 20, 4 (2012)

[17] G. Biavati, G. Di Donfrancesco, F. Cairo, D.G. Feist, Applied Optics 50, 5872 (2011)

[18] A. López-Oramas, Ph.D. thesis, Universitat Autònoma de Barcelona (2014)

[19] M. Gaug et al., in EPJ Web of Conferences (2017), Vol. 144, p. 01010

[20] M. Iarlori et al., The Raman LIDAR for the preproduction phase of CTA, in these proceedings (2018) 